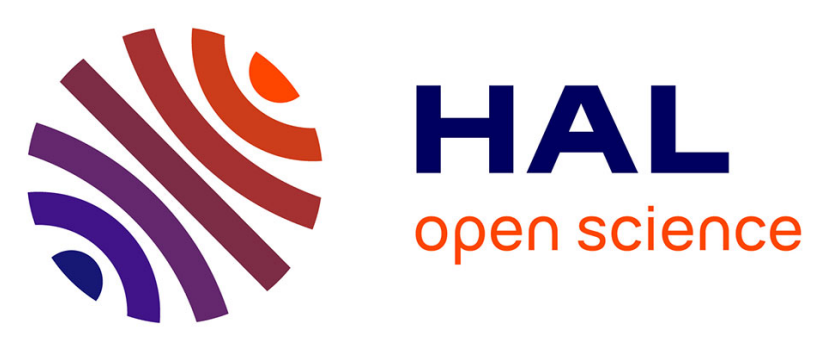

\title{
Asymptotic Modeling of the Axisymmetric Flow of a Thermal Binary Gas Mixture in a Circular Micro-channel
}

\author{
Cédric Croizet, Renée Gatignol
}

\section{To cite this version:}

Cédric Croizet, Renée Gatignol. Asymptotic Modeling of the Axisymmetric Flow of a Thermal Binary Gas Mixture in a Circular Micro-channel. 30th International Symposium on Rarefied Gas Dynamics, Jul 2016, Victoria, BC, Canada. 10.1063/1.4967603 . hal-01445771

\section{HAL Id: hal-01445771 https://hal.sorbonne-universite.fr/hal-01445771}

Submitted on 25 Jan 2017

HAL is a multi-disciplinary open access archive for the deposit and dissemination of scientific research documents, whether they are published or not. The documents may come from teaching and research institutions in France or abroad, or from public or private research centers.
L'archive ouverte pluridisciplinaire HAL, est destinée au dépôt et à la diffusion de documents scientifiques de niveau recherche, publiés ou non, émanant des établissements d'enseignement et de recherche français ou étrangers, des laboratoires publics ou privés. 


\title{
Asymptotic Modeling of the Axisymmetric Flow of a Thermal Binary Gas Mixture in a Circular Micro-channel
}

\author{
Croizet Cédric $^{1, a)}$ and Renée Gatignol ${ }^{1}$ \\ ${ }^{1}$ Sorbonne Universités, UPMC Univ Paris 06, CNRS, UMR 7190, Institut Jean le Rond d'Alembert, F-75005, Paris, \\ France \\ a)cedric.croizet@upmc.fr
}

\begin{abstract}
Sub-millimeter-sized channels are present in many medical and industrial tools such as micro-filters. In order to describe the gas flows in these micro-channels, the DSMC methods are frequently used but a large computation time is usually required to obtain the solutions $[1,2]$. Consequently, it is of main interest to develop alternative methods to describe these flows. In this contribution, we are interested in flows at low Mach numbers and with low to moderate Knudsen numbers so that the flow is in the slip regime. We propose an asymptotic model for the axisymmetric flow of a mixture of two compressible gases in circular microchannels with a temperature gradient at the wall. The model is obtained from the Navier-Stokes-Fourier equations. The results of the model are compared to DSMC simulations and the influence of the temperature gradient which is present along the walls is investigated.
\end{abstract}

\section{INTRODUCTION}

Micro-metric devices are involved in technologies with broad potential application: they are present in various industrial manufacturing processes, medical tools and in measuring instruments. Consequently, a large number of works are devoted to the analysis of gas flows in micro-channels involved in these systems. Various approaches exist: theoretical, numerical and experimental $[1,2,3,4]$. In a numerical point of view, the DSMC methods are very well adapted but they are expensive in computation time [1,2]. Consequently, the development of asymptotic models describing these flows is an interesting alternative. This approach has been used in the case of a single gas in [4, 5] for instance and for a gas mixture in a plane channel [6] or in a cylindrical pipe [7]. In a previous work [8] the mixing length in a micro-channel of two parallel gas streams has been analyzed from DSMC simulations.

In this contribution, our purpose is to model the flow of a mixture of two compressible gases in a circular microchannel. The geometry is showed in Fig. 1. The temperature of the channel wall depends on the longitudinal space variable $z$. The aim is to model these micro-flows with a macroscopic approach and to obtain an asymptotic model for describing the main physical phenomena. The two components are compressible. We are interested in flows at low Mach numbers and at low to moderate Knudsen numbers that corresponds to the slip-flow regime. Consequently, the movement equations are the usual macroscopic balance laws of mass, momentum and energy (Navier-Stokes-Fourier equations) with additional coupling terms in the momentum and energy equations [9, 10, 7]. In this weakly rarefied regime, the usual no-slip wall boundary conditions are replaced with slip conditions. In this paper, first order jump boundary conditions for the velocity and the temperature are written [4] at the walls.

The flow is assumed to be axisymmetric and steady. At the circular channel inlet, each gas has its own pressure and velocity. In the next section we present the model equations. For each gas, the balance equations are written with dimensional and dimensionless quantities. The characteristic velocities in each direction are identical for the two gases. A small parameter $\epsilon$ related to the geometry of the micro-pipe is built from the longitudinal and radial characteristic length scales. We introduce, for each gas, the Mach number and the Reynolds number built with the longitudinal length scale. The Knudsen number can be expressed in terms of these two dimensionless numbers. In the following sections, the solution at the first order in $\epsilon$ is investigated and compared to DSMC simulations in the same configuration. The last section is devoted to a short conclusion. 


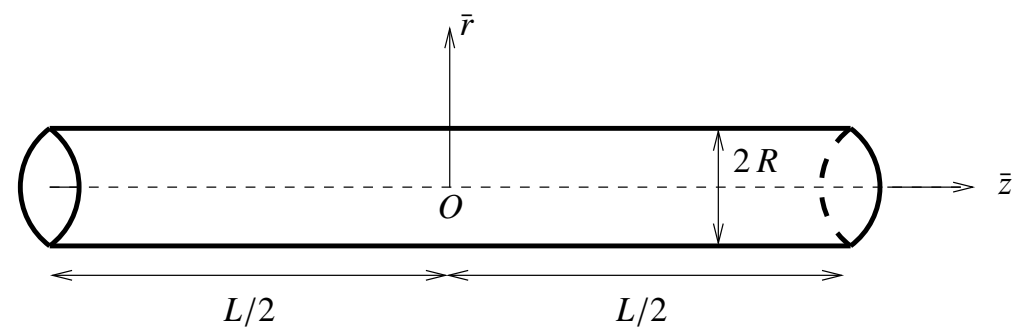

FIGURE 1. Channel geometry

\section{MODEL EQUATIONS}

The micro-channel has the length $L=10 \mu \mathrm{m}$ and the radius $R=1 \mu \mathrm{m}$ (Fig. 1). The channel wall at $\bar{r}=R$ is at rest and has a temperature depending on the longitudinal coordinate $\bar{T}_{w}(\bar{z})$. The movement equations are the Navier-StokesFourier equations for a mixture of two ideal gases denoted with " $a$ " and " $b$ ", with constant heat capacities $c_{p a}$ and $c_{p b}$ at constant pressure and $c_{v a}$ and $c_{v b}$ at constant volume. The shear viscosities $\mu_{a}$ and $\mu_{b}$ and thermal conductivities $\kappa_{a}$ and $\kappa_{b}$ depend on the temperature only and are such as the Prandtl numbers are constant. The model equations are the usual balance equations with additional coupling terms. The "bars" are used for the quantities with dimension except when they are constant. For the species $a$, we have the three following balance equations for the mass, the momentum and the energy:

$$
\begin{gathered}
\frac{\partial \bar{\rho}_{a}}{\partial \bar{t}}+\bar{\nabla} \cdot\left(\bar{\rho}_{a} \overline{\mathbf{u}}_{a}\right)=0 \\
\frac{\partial \bar{\rho}_{a} \overline{\mathbf{u}}_{a}}{\partial \bar{t}}+\bar{\nabla} \cdot\left(\bar{\rho}_{a} \overline{\mathbf{u}}_{a} \overline{\mathbf{u}}_{a}-\bar{\sigma}_{a}\right)=\bar{v}_{a b} \bar{\rho}_{a}\left(\overline{\mathbf{u}}_{a}-\overline{\mathbf{u}}_{a}^{*}\right), \\
\frac{\partial}{\partial \bar{t}}\left(\bar{\rho}_{a} \overline{\mathbf{u}}_{a}^{2}+\bar{\rho}_{a} \bar{e}_{a}\right)+\bar{\nabla} \cdot\left[\bar{\rho}_{a}\left(\frac{1}{2} \overline{\mathbf{u}}_{a} \cdot \overline{\mathbf{u}}_{a}+\bar{e}_{a}\right) \overline{\mathbf{u}}_{a}-\bar{\sigma}_{\mathbf{a}} \cdot \overline{\mathbf{u}}_{\mathbf{a}}+\overline{\mathbf{q}}_{\mathbf{a}}\right]=\bar{S}_{a}^{E} .
\end{gathered}
$$

The equations for the species $b$ are obtained by replacing $a$ with $b$ and vice versa. We introduce the notation " $\theta$ " $(\theta=a, b)$ in order to denote one of the species. Because of the axisymmetry, the velocity field of the gas " $\theta$ " $(\theta=a, b)$ is: $\overline{\mathbf{u}}_{\theta}=\bar{u}_{\theta}(\bar{r}, \bar{z}) \mathbf{e}_{r}+\bar{v}_{\theta}(\bar{r}, \bar{z}) \mathbf{e}_{z}$. As well, $\rho_{\theta}$ is the volumetric mass, $\bar{\sigma}_{\theta}$ is the Navier-Stokes stress tensor of the species $\theta, \overline{\mathbf{q}}_{\theta}$ is the heat flux vector and $\bar{e}_{\theta}$ is the specific internal energy. In addition to the usual equations, some coupling terms appear in the previous equations. These terms are derived from a BGK-type model for binary gas mixture (see for instance $[10,11])$. From this modeling we have:

$$
\begin{gathered}
\overline{\mathbf{u}}_{a}^{*}=\overline{\mathbf{u}}_{b}^{*}=\frac{m_{a} \overline{\mathbf{u}}_{a}+m_{b} \overline{\mathbf{u}}_{b}}{m_{a}+m_{b}}, \\
\bar{v}_{a b}=\bar{v}_{a}\left(\frac{d_{a}+d_{b}}{2 d_{a}}\right)^{2}\left(\frac{m_{a}+m_{b}}{2 m_{b}}\right)^{\frac{1}{2}} \frac{\bar{n}_{b}}{\bar{n}_{a}} \psi_{a b},
\end{gathered}
$$

with:

$$
\psi_{a b}=\left(\left(\frac{d_{a}}{d_{b}}\right)^{2} \sqrt{\frac{m_{b}}{m_{a}}} \frac{\Omega_{b}}{\Omega_{a}} \frac{\bar{\mu}_{a}}{\bar{\mu}_{b}} \frac{\bar{T}_{b}}{\bar{T}_{a}}\right)^{1 / 2}
$$

where $m_{\theta}$ is the molecular mass of the species $\theta$ and where $d_{\theta}, \bar{v}_{\theta}$ and $\bar{n}_{\theta}$ are respectively the molecular diameter, the collision frequency and the numerical density of the species $\theta$. With these definitions, $v_{b a}$ is obtained from $v_{a b}$ by replacing "a" with "b" and the total number of collisions between species $a$ and $b$ is balanced:

$$
\bar{n}_{a} \bar{v}_{a b}=\bar{n}_{b} \bar{v}_{b a} \text {. }
$$

As a consequence, the conservation of the global momentum is satisfied. Additionally:

$$
\begin{aligned}
\bar{v}_{\theta} & =\frac{\bar{p}_{\theta}}{\bar{\mu}_{\theta}} \Omega_{\theta} \quad \text { with } \quad \bar{\mu}_{\theta}=\mu_{\theta}^{r e f}\left(\frac{\bar{T}_{\theta}}{T_{\theta}^{r e f}}\right)^{\omega_{\theta}}, \\
\Omega_{\theta} & =\frac{5\left(\alpha_{\theta}+1\right)\left(\alpha_{\theta}+2\right)}{\alpha_{\theta}\left(7-2 \omega_{\theta}\right)\left(5-2 \omega_{\theta}\right)},
\end{aligned}
$$


where $\bar{p}_{\theta}$ is the pressure of the species $\theta, \alpha_{\theta}$ is a constant depending on the collision potential model and where $\omega_{\theta}$ is the power exponent of the viscosity law (see $[1,10])$. In the energy balance equation the coupling term may be expressed as:

$$
\bar{S}_{a}^{E}=\frac{1}{2} \bar{\rho}_{a} \bar{v}_{a b}\left[\overline{\mathbf{u}}_{a}^{* 2}-\overline{\mathbf{u}}_{a}^{2}+\frac{2 r_{a}}{\gamma_{a}-1}\left(\bar{T}_{a}^{*}-\bar{T}_{a}\right)\right],
$$

with $r_{a}=k / m_{a}$ where $k$ is the Boltzmann constant and where $\bar{T}_{a}$ is the temperature of the species $a$. In this expression, the temperature $\bar{T}_{a}^{*}$ is given by a Morse formula [10] extended to polyatomic ideal gases:

$$
\bar{T}_{a}^{*}=\bar{T}_{a}+\frac{3}{2}\left(\gamma_{a}-1\right) \frac{2 m_{a} m_{b}}{\left(m_{a} m_{b}\right)^{2}}\left(\bar{T}_{b}-\bar{T}_{a}+\frac{m_{b}}{6 k}\left(\overline{\mathbf{u}}_{b}-\overline{\mathbf{u}}_{a}\right)^{2}\right) .
$$

According to this definition and equation (7): $\bar{S}_{a}^{E}+\bar{S}_{b}^{E}=0$ that coincides with energy conservation. In addition, we have the ideal gas equations:

$$
\bar{p}_{\theta}=r_{\theta} \bar{\rho}_{\theta} \bar{T}_{\theta}
$$

and the usual Fourier and Navier-Stokes constitutive laws. For the viscosity coefficients, we adopt the Stokes hypothesis. The first order wall boundary conditions are [3, 4]:

$$
\begin{aligned}
& \left.\bar{v}_{\theta}\right|_{\bar{r}=R}=\left(-\frac{\bar{\mu}_{\theta} \sigma_{\theta p}}{\bar{p}_{\theta}} \sqrt{2 r_{\theta} \bar{T}_{\theta}} \frac{\partial \bar{v}_{\theta}}{\partial \bar{r}}+\frac{\sigma_{\theta T} r_{\theta} \bar{\mu}_{\theta}}{\bar{p}_{\theta}} \frac{\partial \bar{T}_{\theta}}{\partial \bar{z}}\right)_{\bar{r}=R}, \\
& \left.\bar{u}_{\theta}\right|_{\bar{r}=R}=0, \\
& \left.\bar{T}_{\theta}\right|_{\bar{r}=R}=\bar{T}_{w}(\bar{z})-\left(\xi_{\theta T} \sqrt{2 r_{\theta} \bar{T}_{\theta}} \frac{\bar{\mu}_{\theta}}{\bar{p}_{\theta}} \frac{\partial \bar{T}_{\theta}}{\partial \bar{r}}\right)_{\bar{r}=R}
\end{aligned}
$$

where the constant coefficients $\sigma_{\theta p}, \sigma_{\theta T}$ and $\xi_{\theta T}$ characterize the viscous and thermal slip of the species $\theta$ on the walls [12]. The upstream and downstream boundary conditions for the pressures will be specified further.

In order to discuss the magnitude orders of the terms in the model equations, we write the corresponding dimensionless equations. We choose $L$ as the longitudinal length scale and $R$ as the radial length scale and we set $\epsilon=R / L<<1$. Additionally, the characteristic values of the radial and longitudinal velocities, the temperature, the pressure and the volumetric mass are respectively: $U$ and $V, T_{c}$ (for the two species), $p_{\theta c}$ and $\rho_{\theta c}$ (with $p_{\theta c}=r_{\theta} \rho_{\theta c} T_{c}$, $\theta=a, b)$. With these characteristic scales, we built the following dimensionless numbers:

$$
\gamma_{a}=\frac{c_{p a}}{c_{v a}}, \quad \quad \operatorname{Pr}_{a}=\frac{\mu_{a} c_{p a}}{\kappa_{a}}, \quad \quad R e_{a}=\frac{\rho_{a c} V L}{\mu_{a}}, \quad M a_{a}=\frac{V}{\sqrt{\gamma_{a} r_{a} T_{c}}},
$$

Obviously, similar dimensionless numbers are built for the second species. Since $\operatorname{Pr}_{\theta}$ is constant, $\mu_{\theta}$ and $\kappa_{\theta}$ are proportional to $T_{\theta}^{\omega_{\theta}}$. Now we are interested in the steady case. From the Principe of Least Degeneracy [13], we must keep the two terms in the dimensionless mass laws. Consequently $U=\epsilon V$. In order to investigate the significative degeneracies, we assume that the Reynolds number and that the Mach number are small or of order unity; we set $R_{a}=R_{a} \epsilon^{\alpha}$ and $M a_{a}=M_{a} e^{\beta}$ where both $R_{a}$ and $M_{a}$ are of order of the unity. The analysis of the magnitudes of the terms in the dimensionless balance laws derived from (1), (2) and (3) leads to a Mach number of order $\epsilon$ and to a Reynolds number of order unity that is to $\alpha=0$ and $\beta=1$ [11]. At the first approximation order, we have the following dimensionless equations:

$$
\frac{1}{r} \frac{\partial}{\partial r}\left(r \rho_{a} u_{a}\right)+\frac{\partial}{\partial z}\left(\rho_{a} v_{a}\right)=0
$$

for the mass. For the longitudinal and radial balance of momentum, we have:

$$
\begin{gathered}
0=-\frac{1}{\gamma_{a} M_{a}^{2}} \frac{\partial p_{a}}{\partial z}+\frac{1}{R_{a}} \frac{1}{r} \frac{\partial}{\partial r}\left(r \mu_{a} \frac{\partial v_{a}}{\partial r}\right)+F_{a b} \frac{\rho_{b c}}{\rho_{a c}}\left(\frac{\mu_{a c}}{\mu_{b c}}\right)^{\frac{1}{2}} \frac{R_{a}}{\gamma_{a} M_{a}^{2}} p_{a} p_{b} \frac{\left(v_{b}-v_{a}\right)}{T(z)^{1+\frac{\omega_{a}+\omega_{2}}{2}}} \\
0=-\frac{1}{\gamma_{a} M_{a}^{2}} \frac{\partial p_{a}}{\partial r}
\end{gathered}
$$

with:

$$
F_{a b}=\left(\frac{m_{b}}{m_{a}}\right)^{\frac{1}{4}} \frac{m_{a}}{\sqrt{2 m_{b}\left(m_{a}+m_{b}\right)}}\left(\frac{d_{a}+d_{b}}{2 d_{a}}\right)^{2} \frac{d_{a}}{d_{b}} \sqrt{\Omega_{a} \Omega_{b}}
$$


For the species $a$, the first order dimensionless energy balance equation is:

$$
0=\frac{\gamma_{a}}{R_{a} \operatorname{Pr}_{a}}\left[\frac{1}{r} \frac{\partial}{\partial r}\left(r \kappa_{a} \frac{\partial T_{a}}{\partial r}\right)\right]+G_{a b} \frac{R_{a}}{\gamma_{a} M_{a}^{2}} \frac{\rho_{b} p_{a}}{\sqrt{\mu_{a} \mu_{b}}} \sqrt{\frac{T_{b}}{T_{a}}}\left(T_{b}-T_{a}\right),
$$

with:

$$
G_{a b}=\frac{3 m_{a}\left(\gamma_{a}-1\right)}{m_{a}+m_{b}} \frac{\rho_{b c}}{\rho_{a c}} \frac{\mu_{a c}}{\mu_{b c}} F_{a b}
$$

For the sake of simplicity, we do not write here the dimensionless equations for the other species but they are easily obtained by changing $a$ in $b$ and vice versa in the previous equations. First we consider the energy balance equation (21) and we assume that the two gases are in thermal equilibrium. In that case, the equation (21) with the boundary condition (15) admits a unique solution:

$$
T_{a}(r, z)=T_{w}(z)=T_{b}(r, z)=T(z)
$$

Finally, the dimensionless wall boundary conditions for the velocities are:

$$
v_{\theta}(r=1, z)=-K_{\theta} \frac{\left[T_{\theta}(z)\right]^{\omega_{a}+\frac{1}{2}}}{p_{\theta}(z)} \frac{\partial v_{\theta}}{\partial r}(r=1, z)+\frac{\sigma_{\theta T}}{R_{\theta}}\left[T_{\theta}(z)\right]^{\omega_{a}} \frac{d T_{\theta}}{d z}(z) \quad u_{\theta}(r=1, z)=0
$$

with: $K_{\theta}=\sigma_{\theta p} \sqrt{2 \gamma_{\theta}} \frac{M_{\theta}}{R_{\theta}}$. At the first order, we obtain a set of six equations: the mass and the momentum equations for the two species. In the next section we will investigate the solution of this set of equations.

\section{FIRST ORDER SOLUTION}

The radial momentum equations (19) for the two species lead to pressures depending only on the longitudinal space variable $z$. If we use, in addition, the dimensionless ideal gas law, we have: $p_{a}(z)=\rho_{a}(z) T(z)$ and $p_{b}(z)=\rho_{b}(z) T(z)$. Consequently, the problem equations are reduced to:

$$
\begin{aligned}
\frac{1}{r} \frac{\partial}{\partial r}\left(r p_{a} u_{a}\right)+\frac{\partial}{\partial z}\left(p_{a} v_{a}\right) & =0, \\
\frac{1}{r} \frac{\partial}{\partial r}\left(r p_{b} u_{b}\right)+\frac{\partial}{\partial z}\left(p_{b} v_{b}\right) & =0 \\
\frac{1}{r} \frac{\partial}{\partial r}\left(r \frac{\partial v_{a}}{\partial r}\right)-\frac{B_{a}}{A_{a}} p_{a} p_{b}\left(v_{a}-v_{b}\right) & =\frac{1}{A_{a}} \frac{d p_{a}}{d z}, \\
\frac{1}{r} \frac{\partial}{\partial r}\left(r \frac{\partial v_{b}}{\partial r}\right)+\frac{B_{b}}{A_{b}} p_{a} p_{b}\left(v_{a}-v_{b}\right) & =\frac{1}{A_{b}} \frac{d p_{b}}{d z},
\end{aligned}
$$

with:

$$
A_{\theta}=\frac{\gamma_{\theta} M_{\theta}^{2}}{R_{\theta}}\left[T_{\theta}(z)\right]^{\omega_{\theta}} \quad \theta=a, b
$$

and:

$$
B_{a}=R_{a} \frac{\rho_{b c}}{\rho_{a c}} F_{a b} \frac{\mu_{a c}}{\mu_{b c}}\left[T_{a}(z)\right]^{-\left(1+\frac{\omega_{a}+\omega_{b}}{2}\right)}, \quad B_{b}=R_{b} \frac{\rho_{a c}}{\rho_{b c}} F_{b a} \frac{\mu_{b c}}{\mu_{a c}}\left[T_{b}(z)\right]^{-\left(1+\frac{\omega_{a}+\omega_{b}}{2}\right)}
$$

In the expressions (29) and (30), $A_{a}, A_{b}, B_{a}$ and $B_{b}$ are functions depending on $z$. The longitudinal momentum equations (27) and (28) are solved to obtain the longitudinal velocities. They are the solutions of zero order modified Bessel equations. Since the longitudinal velocities of the two gases must be finite at the channel axis $(r=0)$, the Bessel function of the second kind vanishes in the solutions. With the wall boundary conditions (24), these velocities are explicitly given in terms of the pressures:

$$
v_{a}(r, z)=\frac{1}{A_{a} B_{b}+A_{b} B_{a}}\left[A_{b} B_{a} C_{1} I_{o}\left(\lambda_{1} r\right)+\left(A_{a} \frac{d p_{b}}{d z}-A_{b} \frac{d p_{a}}{d z}\right) \frac{A_{b} B_{a} E_{a}}{E p_{a} p_{b}}\right.
$$




$$
\begin{aligned}
+ & \left.\left(B_{a} \frac{p_{b}}{d z}+B_{b} \frac{d p_{a}}{d z}\right)\left(\frac{r^{2}-1}{4}-\frac{K_{b} T^{\omega_{b}+\frac{1}{2}}}{2 p_{b}} \frac{A_{b} B_{a} E_{a}}{E}-\frac{K_{a} T^{\omega_{a}+\frac{1}{2}}}{2 p_{a}} \frac{A_{a} B_{b} E_{b}}{E}\right)\right] \\
+ & {\left[\frac{A_{a} B_{b} E_{b}}{E} \frac{\sigma_{a T}}{R_{a}} T(z)^{\omega_{a}}+\frac{A_{b} B_{a} E_{a}}{E} \frac{\sigma_{b T}}{R_{b}} T(z)^{\omega_{b}}\right] \frac{d T}{d z}(z), } \\
v_{b}(r, z) & =\frac{1}{A_{a} B_{b}+A_{b} B_{a}}\left[-A_{a} B_{b} C_{1} I_{o}\left(\lambda_{1} r\right)+\left(A_{b} \frac{d p_{a}}{d z}-A_{a} \frac{d p_{b}}{d z}\right) \frac{A_{a} B_{b} E_{b}}{E p_{a} p_{b}}\right. \\
& \left.+\left(B_{a} \frac{p_{b}}{d z}+B_{b} \frac{d p_{a}}{d z}\right)\left(\frac{r^{2}-1}{4}-\frac{K_{b} T^{\omega_{b}+\frac{1}{2}}}{2 p_{b}} \frac{A_{b} B_{a} E_{a}}{E}-\frac{K_{a} T^{\omega_{a}+\frac{1}{2}}}{2 p_{a}} \frac{A_{a} B_{b} E_{b}}{E}\right)\right] \\
+ & {\left[\frac{A_{a} B_{b} E_{b}}{E} \frac{\sigma_{a T}}{R_{a}} T(z)^{\omega_{a}}+\frac{A_{b} B_{a} E_{a}}{E} \frac{\sigma_{b T}}{R_{b}} T(z)^{\omega_{b}}\right] \frac{d T}{d z}(z), }
\end{aligned}
$$

where $I_{o}$ is the first kind modified Bessel function of order zero. Moreover, the following functions depending on $z$ appear in (31) and (32):

$$
\begin{aligned}
\lambda_{1}(z) & =\sqrt{\left(\frac{B_{a}}{A_{a}}+\frac{B_{b}}{A_{b}}\right) p_{a}(z) p_{b}(z)}, \\
E_{\theta}(z) & =I_{o}\left(\lambda_{1}(z)\right)+\frac{K_{\theta} T(z)^{\omega_{\theta}+\frac{1}{2}}}{p_{\theta}(z)} \lambda_{1}(z) I_{1}\left(\lambda_{1}(z)\right) \quad \theta=a, b \\
E(z) & =A_{a} B_{b} E_{b}(z)+A_{b} B_{a} E_{a}(z), \\
C_{1}(z) & =\frac{B_{b}}{E(z)}\left[\frac{A_{b}}{B_{b} p_{a}(z) p_{b}(z)}+\frac{K_{b} T^{\omega_{b}+\frac{1}{2}}}{2 p_{b}(z)}-\frac{K_{a} T^{\omega_{a}+\frac{1}{2}}}{2 p_{a}(z)}\right] \frac{d p_{a}}{d z}(z) \\
& +\frac{B_{a}}{E(z)}\left[-\frac{A_{a}}{B_{a} p_{a}(z) p_{b}(z)}+\frac{K_{b} T^{\omega_{b}+\frac{1}{2}}}{2 p_{b}(z)}-\frac{K_{a} T^{\omega_{a}+\frac{1}{2}}}{2 p_{a}(z)}\right] \frac{d p_{b}}{d z}(z) \\
& +\frac{A_{a} B_{b}+A_{b} B_{a}}{E}\left(\frac{\sigma_{a T}}{R_{a}} T(z)^{\omega_{a}}-\frac{\sigma_{b T}}{R_{b}} T(z)^{\omega_{b}}\right) \frac{d T}{d z}(z),
\end{aligned}
$$

where $I_{1}$ is the first kind modified Bessel function of order one and where $K_{\theta}, A_{\theta}$ and $B_{\theta}(\theta=a, b)$ are given in (24), (29) and (30).

As a consequence of the mass balance equations, the pressure of each gas is given by an ordinary differential equation. In order to obtain these equations, we integrate the mass balance equations of each species (25) and (26) from $r=0$ to $r=1$. We use the wall boundary conditions for the transversal velocities and we obtain:

$$
\begin{aligned}
& \int_{0}^{1} \rho_{a}(z) v_{a}(r, z) r d r=\frac{Q_{m a}}{2}, \\
& \int_{0}^{1} \rho_{b}(z) v_{b}(r, z) r d r=\frac{Q_{m b}}{2},
\end{aligned}
$$

where $Q_{m a}$ and $Q_{m b}$ are the constant dimensionless mass flow rate of each species $\left(Q_{m \theta}=Q_{m \theta}^{*} /\left(\rho_{\theta c} \pi R^{2} V\right), \theta=a, b\right)$. The equations (37) and (38) are integrated in $r$ and then we obtain two ordinary differential equations for the two pressures $p_{a}(z)$ and $p_{b}(z)$. After a few calculations, these equations are written as:

$$
\begin{aligned}
X_{a} \frac{d p_{a}}{d z}+Y_{a} \frac{d p_{b}}{d z} & =\left(A_{a} B_{b}+A_{b} B_{a}\right)\left[\frac{T Q_{m a}}{p_{a}}-\left(\frac{A_{a} B_{b} E_{b} \sigma_{a T}}{E R_{a}} T^{\omega_{a}}+\frac{A_{b} B_{a} E_{a} \sigma_{b T}}{E R_{b}} T^{\omega_{b}}\right) \frac{d T}{d z}\right. \\
& \left.-\frac{2 A_{b} B_{a}}{E \lambda_{1}} I_{1}\left(\lambda_{1}\right)\left(\frac{\sigma_{a T}}{R_{a}} T^{\omega_{a}}-\frac{\sigma_{b T}}{R_{b}} T^{\omega_{b}}\right) \frac{d T}{d z}\right], \\
Y_{b} \frac{d p_{a}}{d z}+X_{b} \frac{d p_{b}}{d z} & =\left(A_{a} B_{b}+A_{b} B_{a}\right)\left[\frac{T Q_{m b}}{p_{b}}-\left(\frac{A_{a} B_{b} E_{b} \sigma_{a T}}{E R_{a}} T^{\omega_{a}}+\frac{A_{b} B_{a} E_{a} \sigma_{b T}}{E R_{b}} T^{\omega_{b}}\right) \frac{d T}{d z}\right. \\
& \left.+\frac{2 A_{a} B_{b}}{E \lambda_{1}} I_{1}\left(\lambda_{1}\right)\left(\frac{\sigma_{a T}}{R_{a}} T^{\omega_{a}}-\frac{\sigma_{b T}}{R_{b}} T^{\omega_{b}}\right) \frac{d T}{d z}\right],
\end{aligned}
$$



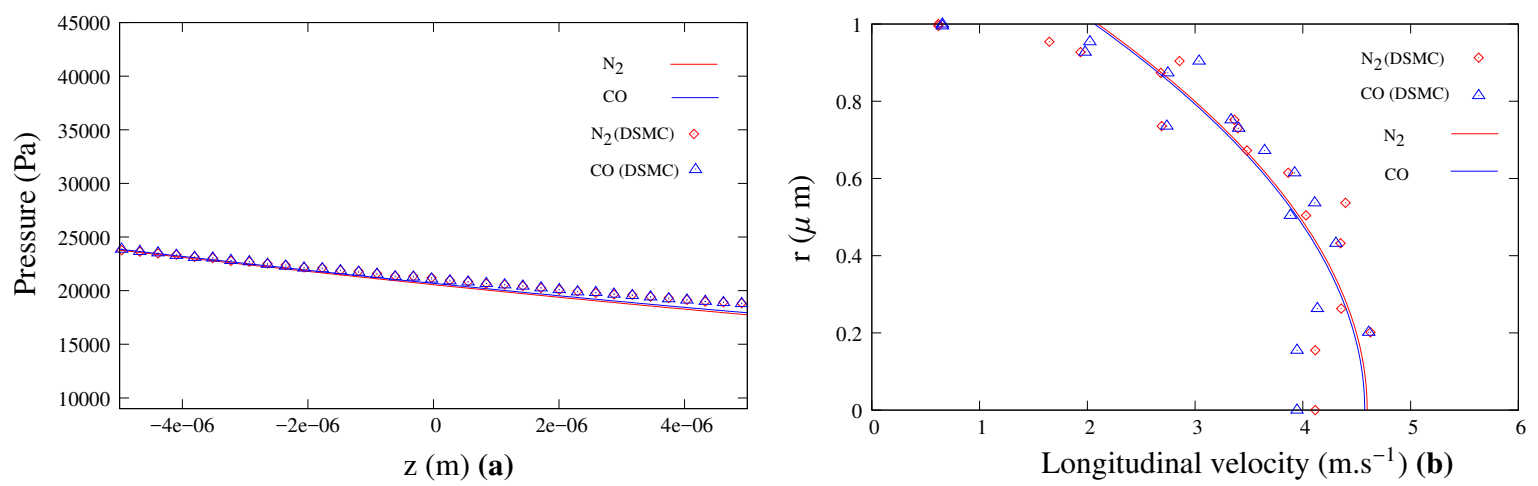

FIGURE 2. Comparison to DSMC: Pressures along the micro-channel axis (a) - Velocity profiles at the channel outlet (b)

with:

$$
\begin{aligned}
X_{a} & =\frac{A_{b} B_{a} B_{b}}{E \lambda_{1}}\left[\frac{2 A_{b}}{B_{b} p_{a} p_{b}}+\frac{K_{b} T^{\omega_{b}+\frac{1}{2}}}{p_{b}}-\frac{K_{a} T^{\omega_{a}+\frac{1}{2}}}{p_{a}}\right] I_{1}\left(\lambda_{1}\right)-\frac{A_{b}^{2} B_{a} E_{a}}{E p_{a} p_{b}} \\
& -B_{b}\left[\frac{1}{8}+\frac{K_{b} T^{\omega_{b}+\frac{1}{2}}}{2 p_{b}} \frac{A_{b} B_{a} E_{a}}{E}+\frac{K_{a} T^{\omega_{a}+\frac{1}{2}}}{2 p_{a}} \frac{A_{a} B_{b} E_{b}}{E}\right], \\
Y_{a} & =+\frac{A_{b} B_{a}^{2}}{E \lambda_{1}}\left[-\frac{2 A_{a}}{B_{a} p_{a} p_{b}}+\frac{K_{b} T^{\omega_{b}+\frac{1}{2}}}{p_{b}}-\frac{K_{a} T^{\omega_{a}+\frac{1}{2}}}{p_{a}}\right] I_{1}\left(\lambda_{1}\right)+\frac{A_{a} A_{b} B_{a} E_{a}}{E p_{a} p_{b}} \\
& -B_{a}\left[\frac{1}{8}+\frac{K_{b} T^{\omega_{b}+\frac{1}{2}}}{2 p_{b}} \frac{A_{b} B_{a} E_{a}}{E}+\frac{K_{a} T^{\omega_{a}+\frac{1}{2}}}{2 p_{a}} \frac{A_{a} B_{b} E_{b}}{E}\right] .
\end{aligned}
$$

The expressions of $X_{b}$ and $Y_{b}$, respectively, are obtained from $X_{a}$ and $Y_{a}$ by changing $a$ in $b$ and vice versa. The equations (39) and (40) are coupled and linear in term of the longitudinal pressure gradients but non linear in $z$.

\section{NUMERICAL SOLUTIONS}

In order to solve the two equations (39) and (40), we need the wall temperature and four boundary conditions: for instance, the pressures $p_{a}$ and $p_{b}$ at the channel inlet and the dimensionless mass flow rates $Q_{m a}$ and $Q_{m b}$. These values may be set from DSMC simulation in the same geometry. We have: $\bar{p}_{a}(-L / 2)=2.37810^{4} \mathrm{~Pa}, \bar{p}_{b}(-L / 2)=$ $2.38710^{4} \mathrm{~Pa}, \bar{Q}_{m a}=7.86110^{-13} \mathrm{~kg} \cdot \mathrm{m}^{-1} \cdot \mathrm{s}^{-1}$ and $\bar{Q}_{m b}=7.88110^{-13} \mathrm{~kg} \cdot \mathrm{m}^{-1} \cdot \mathrm{s}^{-1}$. The wall temperature decreases linearly from $300 \mathrm{~K}$ to $200 \mathrm{~K}$. From DSMC simulations, we set equally the characteristic values of the longitudinal velocity and of the pressures: $V=1 \mathrm{~m} \cdot \mathrm{s}^{-1}$ and $p_{a c}=p_{b c}=10^{5} \mathrm{~Pa}$. In addition, we have to set the values of the various constants related to the nature of the gases. We are interested in gases with the same molecular mass so we assume that the species $a$ is nitrogen and that the species $b$ is Carbon monoxide. Consequently, from [1], we have: $m_{a}=m_{b}=46.510^{-27} \mathrm{~kg}, d_{a}=4.1110^{-10} \mathrm{~m}, \gamma_{a}=\gamma_{b}=1.4,\left[\mu_{a}\right]_{V S S}=1.72510^{-5}$ Pa.s, $\alpha_{a}=1.36, \omega_{a}=0.74$, $r_{a}=r_{b}=296.77 \mathrm{~J} \cdot \mathrm{kg}^{-1} \cdot \mathrm{K}^{-1}, d_{b}=4.1210^{-10} \mathrm{~m},\left[\mu_{b}\right]_{V S S}=1.70210^{-5}$ Pa.s, $\alpha_{b}=1.49$ and $\omega_{b}=0.73$. These values are those used in the DSMC simulations with Bird's DS2V program [14]. The two equations (39) and (40) are solved with MATLAB using a variable step Runge-Kutta method of fourth order. With these solutions for the pressures, the longitudinal velocities are computed from (31) and (32). The numerical solutions of the analytical model are compared to the results of the DSMC simulations in Fig. 2. Obviously, the computation time is strongly decreased for the asymptotic model ; while DSMC need a few days to produce a solution, only a few seconds are required for our model. We have a good agreement between the DSMC simulation and the asymptotic solution for the pressure of the two species. Since the pressure difference between the inlet and the outlet of the channel, for the two species, is weak, the pressures, in both calculations, seem to be quasi linear in $z$. Nevertheless the pressures are underestimated at the channel outlet by our model: the difference is about $4 \%$. This difference between the asymptotical appproach and DSMC simulations is a consequence of the strong non linearity of the equations (39) and (40): They are very sensitive to the boundary conditions. As a consequence, a small variation in the inlet pressure and in the mass flow rate leads 




FIGURE 3. Velocity profiles at different channel sections

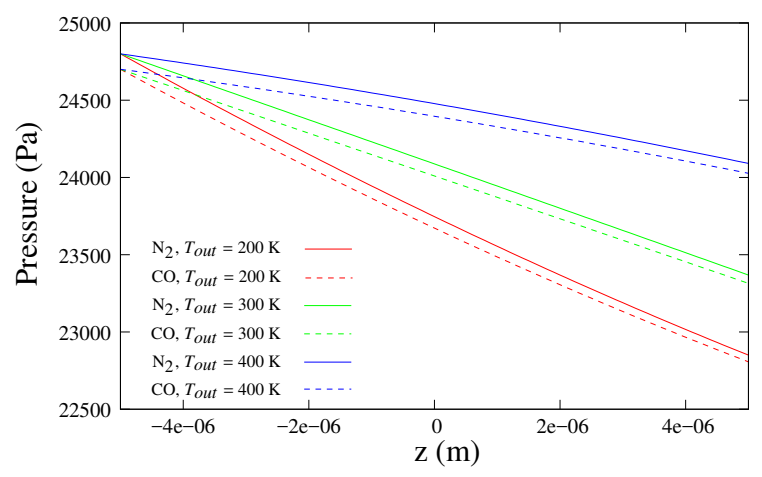

FIGURE 4. Pressure (Pa) along the channel axis with $T_{\text {in }}=300 \mathrm{~K}$ and different values for $T_{\text {out }}$

to a great variation of the solution. In addition, these boundary conditions are obtained from DSMC simulations. Consequently, because of the numerical noise in the DSMC results, it is not easy to set the boundary conditions of the analytical set of equations at a convenient level of accuracy. Moreover, the statistical error for the velocities in DSMC computations is inversely proportional to the Mach number [15] which is of order $2.10^{-2}$ in the present case. The result is an important DSMC error on the velocities (of order 2\%) and, consequently, on the mass flow rate. In order to limit the effects of this error, we set the mass flow rate to the mean of three values calculated in different sections of the channel.

The evolution of the longitudinal velocities is plotted on Fig. 3. We denote a small difference for the two species. This is explained by the differences in the two gases properties and by the difference of inlet conditions. As an effect of the thermal creep, we observe that the longitudinal velocities decrease with the pressures along the micro-channel.

For the same gases, we focus on the effect of the constant gradient of temperature. The inlet temperature is still $300 \mathrm{~K}$ and we set different values of the outlet temperature. The boundary conditions are: $\bar{p}_{\text {ain }}=2.4810^{4} \mathrm{~Pa}$, $\bar{p}_{\text {bin }}=2.4710^{4} \mathrm{~Pa}, \bar{Q}_{m a}=1.86610^{-13} \mathrm{~kg} \cdot \mathrm{m}^{-1} \cdot \mathrm{s}^{-1}$ and $\bar{Q}_{m b}=1.84510^{-13} \mathrm{~kg} \cdot \mathrm{m}^{-1} \cdot \mathrm{s}^{-1}$. Figure 4 shows the evolution of the pressure of each species for three different outlet temperatures. The pressures decrease with $z$. For a given value of $z$, the pressures increase with the outlet temperature. This result is in agreement with the observations in the plane case [6]. The velocity profiles at the channel inlet and outlet are plotted in Fig. 5. In the isothermal case, as expected [7], the longitudinal velocity increases along the channel as the pressure decreases. In the non isothermal cases, the effect of the thermal creep is observed. It induces a movement of the gases from the cold end of the channel to the hot one: when the inlet temperature is greater than the outlet temperature (case $T_{\text {out }}=200 \mathrm{~K}$ on Fig. 5), the effects of the pressure gradient and of the temperature gradient are opposite and, as a consequence, we observe that the velocity decreases along the channel. When the inlet temperature is lower than the outlet temperature, both the pressure and temperature gradients accelerate the gases along the channel. 

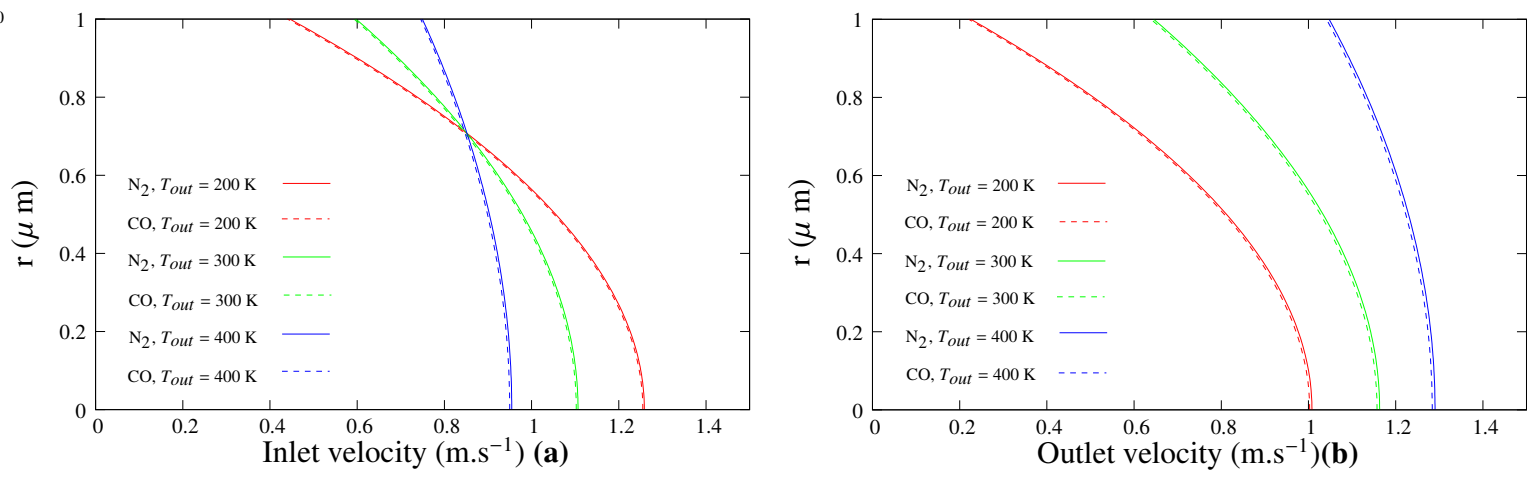

FIGURE 5. Longitudinal velocities at the channel inlet (a) and outlet (b) for $T_{\text {in }}=300 \mathrm{~K}$ and different values for $T_{\text {out }}$

\section{CONCLUSION}

In this contribution, we present an original asymptotic study to analyze the flow of a gaseous mixture in a microchannel. The model is obtained from Navier-Stokes-Fourier equations with additional coupling terms. The longitudinal velocities are given explicitly in term of the pressures that are computed from a first order set of differential equations. The effect of the wall temperature gradient has been investigated. The advantage of this method, compared to DSMC simulations, is a very short computation time.

The results have been compared to DSMC simulations and the two methods are in good agreement. The main difficulty in comparing the two methods is to set the correct boundary conditions for the analytical model which is strongly non linear. This non linearity comes from the coupling terms. In the future, less penalizing coupling terms could be investigated, in the case of gases with very different molecular masses for instance.

\section{REFERENCES}

[1] G. A. Bird, "Gas dynamics and the direct simulation of gas flows," (Clarendon Press, 1994).

[2] O. Aktas, N. R. Aluru, and U. Ravaioli, J. Microelectromech. S. 10, 538-549 (2001).

[3] S. Kandlikar, S. Garimella, D. Li, S. Colin, and M. King, "Transfer and fluid flow in minichannels and microchannels," (Elsevier, 2005).

[4] G. Karniadakis and A. Beskok, "Microflows - fundamentals and simulation," (Springer, 2006).

[5] R. Gatignol and C. Croizet, "Asymptotic modelling of the flows in micro-channel by using macroscopic balance equation," in Proceedings of the 27th Int. Symp. on Rarefied Gas Dynamics, edited by A. Levin, I. J. Wysong, and A. L. Garcia (American Institute of Physics, New York, 2011), pp. 730-735.

[6] R. Gatignol and C. Croizet, "Asymptotic modelling of the axisymmetric flow of a binary gas mixture in a circular microchannel," in Proceedings of the 28th Int. Symp. of Rarefied Gas Dynamics, AIP Conference Proceedings (American Institute of Physics, New York, 2014), pp. 807-814.

[7] C. Croizet and R. Gatignol, "Asymptotic modelling of the axisymmetric flow of a binary gas mixture in a circular microchannel," in Proceedings of the 28th Int. Symp. of Rarefied Gas Dynamics, AIP Conference Proceedings (American Institute of Physics, New York, 2014), pp. 799-806.

[8] M. Reyhanian, C. Croizet, and R. Gatignol, Mechanics and Industry 14, 453-460 (2013).

[9] S. Chapman and T. G. Cowling, "The mathematical theory of non-uniform gases," (C. U. P., 1952), pp. 278-294.

[10] T. G. Elizarova, I. A. Graur, and J. C. Lengrand, Eur. J. Mech. B-Fluids 3, 351-369 (2001).

[11] M. Reyhanian-Mashhadi, "Simulation numérique par la méthode de monte carlo (dsmc) et modélisation analytique d'un mélange gazeux dans un micro canal," Ph.D. thesis, Université Pierre et Marie Curie, Paris 2011.

[12] F. Sharipov and V. Seleznev, J. Phys. Chem. Ref. Data 27, 657-706 (1998).

[13] M. Van Dyke, "Perturbation methods in fluid mechanic," (Academic Press, 1964).

[14] G. M. Bird, "Dsmc resources," (2014).

[15] N. Hadjiconstantinou, A. Garcia, and G. Bazant, J. Comp. Phys. 187, 274-297 (2003). 